\title{
Second-harmonic patterned polarization-analyzed reflection confocal microscope
}

Chukwuemeka Okoro

Kimani C. Toussaint, Jr. 


\title{
Second-harmonic patterned polarization-analyzed reflection confocal microscope
}

\author{
Chukwuemeka Okoro ${ }^{a}$ and Kimani C. Toussaint Jr.,c,* \\ aUniversity of Illinois at Urbana-Champaign, PROBE Lab, Department of Electrical and Computer Engineering, Urbana, Illinois, United States \\ bUniversity of Illinois at Urbana-Champaign, PROBE Lab, Department of Mechanical Science and Engineering, Urbana, Illinois, United States \\ 'University of Illinois at Urbana-Champaign, PROBE Lab, Affiliate in the Department of Electrical and Computer Engineering and Bioengineering, \\ Urbana, Illinois, United States
}

\begin{abstract}
We introduce the second-harmonic patterned polarization-analyzed reflection confocal (SPPARC) microscope-a multimodal imaging platform that integrates Mueller matrix polarimetry with reflection confocal and second-harmonic generation (SHG) microscopy. SPPARC microscopy provides label-free three-dimensional (3-D), SHG-patterned confocal images that lend themselves to spatially dependent, linear polarimetric analysis for extraction of rich polarization information based on the Mueller calculus. To demonstrate its capabilities, we use SPPARC microscopy to analyze both porcine tendon and ligament samples and find differences in both circular degree-of-polarization and depolarization parameters. Moreover, using the collagen-generated SHG signal as an endogenous counterstain, we show that the technique can be used to provide 3-D polarimetric information of the surrounding extrafibrillar matrix plus cells or EFMC region. The unique characteristics of SPPARC microscopy holds strong potential for it to more accurately and quantitatively describe microstructural changes in collagen-rich samples in three spatial dimensions. ๑ 2017 Society of Photo-Optical Instrumentation Engineers (SPIE) [DOI: 10.1117/1.JBO.22.8.086007]
\end{abstract}

Keywords: confocal microscopy; second-harmonic generation; polarimetry; Mueller matrix; extrafibrillar matrix.

Paper 170425R received Jun. 30, 2017; accepted for publication Jul. 31, 2017; published online Aug. 23, 2017.

\section{Introduction}

Collagen is the most prevalent protein in the human body, ${ }^{1,2}$ and as part of the extracellular matrix (ECM), it plays an important role in regulation of cell behavior. ${ }^{3}$ For type I fibrillar collagen, properties such as alignment, structural organization, and polarization response have been shown to affect and/or depend on tumor progression, ${ }^{4}$ bone development, ${ }^{5}$ aging, and wound healing. ${ }^{6}$ It has even been suggested that part of the embedding ground matrix having little fiber content (called the extrafibrillar matrix or EFM) affects degeneration remodeling ${ }^{7}$ and mechanical behavior. ${ }^{8}$ Hence, quantification of collagen and the surrounding EFM plus cell (EFMC) properties can provide a framework for objective assessment of tissues, and for more accurately monitoring sensitive changes. ${ }^{9-11}$

Second-harmonic generation (SHG) microscopy is a prevalent fibrillar collagen-imaging modality on which quantitative techniques have been applied. ${ }^{12-14}$ SHG microscopy is useful due to its specificity to the noncentrosymmetric structure of collagen, and the technique's optical sectioning and label-free capabilities. ${ }^{15}$ An example of a quantitative approach employed on SHG is the forward-to-backward SHG measurement, which has been used as a potential marker for tendon hydration levels. ${ }^{16}$ Also, Fourier-transform SHG has been used to quantify two-dimensional (2-D) $)^{17,18}$ and three-dimensional (3-D) ${ }^{19}$ collagen fiber spatial organization in tendon.

Quantification of the polarization properties of light scattered from tissues has been exploited as a means to assess collagen. ${ }^{20-22}$ A polarization-based technique able to implement quantitative

*Address all correspondence to: Kimani C. Toussaint, Jr., E-mail: ktoussai@ illinois.edu measures is polarized light microscopy (PLM) ${ }^{23,24}$ PLM leverages the birefringent properties of collagen to increase image contrast, and measure fibril orientation and parallelism. ${ }^{25,26}$ For example, quantitative PLM has been used to measure collagen anisotropy in sheep tendon. ${ }^{27}$ However, PLM is limited to 2-D analysis.

Another group of polarization-based approaches for characterizing collagen invokes the derivation of the second-order polarization susceptibility $\mathbf{M}=\left(\begin{array}{llll}\mathbf{S}_{\mathrm{o} 1} & \mathbf{S}_{\mathrm{o} 2} & \mathbf{S}_{\mathrm{o} 3} & \mathbf{S}_{\mathrm{o} 4}\end{array}\right)\left(\begin{array}{llll}\mathbf{S}_{\mathrm{i} 1} & \mathbf{S}_{\mathrm{i} 2} & \mathbf{S}_{\mathrm{i} 3} & \mathbf{S}_{\mathrm{i} 4}\end{array}\right)^{-1}$ via its relation to the measured SHG intensity. ${ }^{28-30}$ This approach has been employed in revealing significant differences in the susceptibility matrix elements between human melanoma tumor and comparable normal mouse skin tissue, ${ }^{28}$ and for mapping anisotropy information in collagen-muscle junction of chicken wing. ${ }^{31}$ In another study, our group showed the potential of using normalized $\chi^{(2)}$ matrix elements to infer molecular differences in breast biopsy collagen samples. ${ }^{30}$ One shortcoming of this method, however, is the need to assume a model crystallographic arrangement and subsequently fit the measured SHG signal data to this model.

A more comprehensive polarization-based approach entails the use of Mueller matrix polarimetry. This technique invokes the rich and comprehensive Mueller matrix framework for characterization of acquired tissue images. ${ }^{32}$ The Mueller matrices can be decomposed to extract parameters that are representative of the polarization properties of the sample; a popular decomposition method is the Lu-Chipman Mueller matrix polar decomposition (MMPD). ${ }^{33}$ The MMPD, which assumes multiplicative polarization effects in optical systems, has been applied to describe the polarization response of fibrillar collagen ${ }^{34}$ and

$1083-3668 / 2017 / \$ 25.00$ @ 2017 SPIE 
to identify differentiating features between healthy tissues and cancerous human basal cell carcinoma. ${ }^{35}$ Our group also previously applied an adaptation of Mueller matrix polarimetry to SHG microscopy for assessment of collagenous porcine tissue. ${ }^{36}$ Using this adaptation, based on a generalized StokesMueller formalism ${ }^{37-39}$ and particular to two-photon processes, ${ }^{40,41}$ we derived a bimodal mean difference metric $\left(\mu_{\mathrm{d}}\right)$ associated with the degree-of-polarization-which showed increasing variation in porcine tissue with sample thickness. A limitation of this second-order Mueller matrix theory is that the parameters are not as intuitive as the conventional polarimetry case, which is important for understanding the underlying tissue properties.

In general, Mueller matrix polarimetry has been carried out using several image acquisition options, namely, brightfield microscopy, ${ }^{42}$ optical coherence tomography (OCT), ${ }^{43}$ and laser scanning reflectance confocal microscopy. ${ }^{44}$ Polarimetry on OCT is part of the broader polarization-sensitive OCT (PS-OCT) family that has been used to monitor birefringence due to thermal damage in porcine tendon, ${ }^{45}$ quantify birefringent properties of skin, ${ }^{46}$ and assess coronary plaque collagen content. ${ }^{47}$ It has also been combined with SHG for imaging collagen in skin samples from salmon. ${ }^{48}$ PS-OCT has the advantage of 3-D sectioning and deep penetration due to the coherencegating effect and near-IR wavelengths typically used, respectively. ${ }^{49}$ However, in comparison to confocal imaging ${ }^{50}$ and SHG microscopy, PS-OCT generally does not achieve submicron resolution. ${ }^{51}$ In addition, PS-OCT specificity to collagen is not as high as SHG microscopy. ${ }^{52}$ Laser scanning reflectance confocal microscopy presents an attractive polarimetric image acquisition option for several reasons. First, it also enables 3-D optical sectioning. ${ }^{53}$ Furthermore, it is readily used for microscopy of unstained tissue samples. ${ }^{54,55}$ In previous studies, Mueller matrix polarimetry has been combined with a confocal imaging system to obtain 3-D polarization information for collagenous tissues. ${ }^{21,44}$ However, confocal microscopy has no inherent imaging specificity to collagen, and hence, any subsequent analysis based on acquired images cannot discriminate between collagenous and noncollagenous regions. There is, therefore, a need to specify collagen-rich regions with a complementary technique so as to accurately target and characterize the collagen response in tissue samples.

We present herein the second-harmonic patterned polarization-analyzed reflection confocal (SPPARC) microscope, which provides both SHG and linear polarimetric confocal microscopy images at a target imaging plane in a volume. The primary aim of this work is to demonstrate the capabilities of SPPARC microscopy in obtaining spatially dependent polarization information in 3-D, from both collagen fibers in tissue and the surrounding EFMC. It combines the advantage of collagen-specificity from SHG microscopy with the potential insight acquired from linear polarimetry in reflection confocal microscopy. Moreover, the optical sectioning capability of both techniques is retained. The SHG images are used as an endogenous mask to pattern the confocal images, and the resulting collagen-filtered confocal images undergo MMPD analysis for extraction of traditional, linear polarimetric measures, namely, depolarization, retardance, and diattenuation matrices, along with their associated scalars. In addition, the noncollagenous regions comprising the EFMC can also be obtained from the SPPARC images and analyzed. To our knowledge, this is the first time that a single microscopy platform can provide label-free, quantitative information of both the collagen fibers comprising the ECM and the EFMC environment around the fibers.

The paper is organized as follows: we first briefly review the relevant Mueller matrix theory and polar decomposition in Sec. 2. Section 3 describes the experimental setup and sample preparation process, while the results are presented in Sec. 4. In Sec. 5, we discuss and summarize these results.

\section{Theory}

In this section, we review the theory behind Mueller matrix decomposition. The Mueller matrix formalism deals with intensities rather than fields, ${ }^{56}$ and this enables a mathematical description that accommodates the depolarization phenomena. A Mueller matrix linearly relates an input to an output Stokes vector via matrix multiplication given by

$\mathbf{S}_{\mathrm{o}}=\mathbf{M S} \mathbf{S}_{\mathrm{i}}$

where $\mathbf{M}=\left(\begin{array}{llll}m_{00} & m_{01} & m_{02} & m_{03} \\ m_{10} & m_{11} & m_{12} & m_{13} \\ m_{20} & m_{21} & m_{22} & m_{23} \\ m_{30} & m_{31} & m_{32} & m_{33}\end{array}\right)$ is a $4 \times 4$ Mueller matrix and $\mathbf{S}_{\mathrm{i}}$ and $\mathbf{S}_{\mathrm{o}}$ are the input and output Stokes vectors, respectively. To obtain representative Mueller matrices of tissues, multiple Stokes vector measurements should be taken. For a given set of input Stokes vectors and a minimum of four independent output Stokes vector measurements, $\mathbf{M}$ can be uniquely determined by concatenating the vectors into matrices and inverting such that

$\mathbf{M}=\left(\begin{array}{llll}\mathbf{S}_{\mathrm{o} 1} & \mathbf{S}_{\mathrm{o} 2} & \mathbf{S}_{\mathrm{o} 3} & \mathbf{S}_{\mathrm{o} 4}\end{array}\right)\left(\begin{array}{llll}\mathbf{S}_{\mathrm{i} 1} & \mathbf{S}_{\mathrm{i} 2} & \mathbf{S}_{\mathrm{i} 3} & \mathbf{S}_{\mathrm{i} 4}\end{array}\right)^{-1}$.

Due to noise in measurements, it becomes desirable to "oversample" by taking more measurements, and then use the least squares estimate of the inverse (Chapter 22 of Ref. 57)

$\mathbf{M}=\left(\begin{array}{lll}\mathbf{S}_{\mathrm{o} 1} & \cdots & \mathbf{S}_{\mathrm{on}}\end{array}\right)\left(\mathbf{S}_{\mathrm{i} 1} \cdots \mathbf{S}_{\mathrm{in}}\right)_{P}^{-1}$,

where ()$_{P}^{-1}$ represents the pseudoinverse operator and $n(>4)$ is the number of polarization state generator (PSG) configurations used in the measurements.

MMPD decomposes the derived Mueller matrices into three matrices: the diattenuation, retardance, and depolarization matrices $^{33}$ such that

$\mathbf{M}=\mathbf{M}_{\mathbf{\Delta}} \mathbf{M}_{\mathrm{R}} \mathbf{M}_{\mathrm{D}}$

where

$$
\mathbf{M}_{\Delta}=\left(\begin{array}{cc}
1 & \overrightarrow{0}^{T} \\
\vec{P}_{\Delta} & \mathbf{m}_{\Delta}
\end{array}\right), \quad \mathbf{M}_{\mathrm{R}}=\left(\begin{array}{cc}
1 & \overrightarrow{0}^{T} \\
\overrightarrow{0} & \mathbf{m}_{\mathrm{R}}
\end{array}\right), \quad \mathbf{M}_{\mathrm{D}}=\left(\begin{array}{cc}
1 & \vec{D}^{T} \\
\vec{D} & \mathbf{m}_{\mathrm{D}}
\end{array}\right),
$$

where $\mathbf{m}_{\Delta}, \mathbf{m}_{\mathrm{R}}$, and $\mathbf{m}_{\mathrm{D}}$ are the $3 \times 3$ depolarization, retardance, and diattenuation submatrices, respectively, $\vec{P}_{\Delta}$ is the polarizance vector, and $\vec{D}$ is the diattenuation vector. Scalar parameters, derived from the full matrix and submatrices, offer quantitative insight of physically relatable metrics, which are more intuitive for description of the systems. Table 1 lists some common scalar parameters and their respective definitions that can be extracted using MMPD analysis. These parameters 
Table 1 Description of selected scalar parameters obtainable from the submatrix decomposition of the Mueller matrix (first two) and directly from the Mueller matrix (last three). ${ }^{33,58}$

\begin{tabular}{|c|c|c|}
\hline Scalar metric & Mathematical definition & Description \\
\hline Diattenuation & $D=\operatorname{norm}(\vec{D})$ & $\begin{array}{l}\text { Measure of the dependence of the transmittance of the system on incident polarization. } \\
\text { Obtained from the diattenuation vector. }\end{array}$ \\
\hline Depolarization & $\Delta=1-\frac{\left|\operatorname{Tr}\left(\mathbf{M}_{\mathrm{R}}\right)-1\right|}{3}$ & $\begin{array}{l}\text { Measure of depolarization power of the depolarizer matrix on a polarized incident source. } \\
\text { Obtained from the depolarization matrix. }\end{array}$ \\
\hline Linear retardance & $\begin{array}{l}R_{\mathrm{L}}=\cos ^{-1}\left\{\left[\left(m_{R 11}+m_{R 22}\right)^{2}\right.\right. \\
\left.\left.+\left(m_{R 11}+m_{R 22}\right)^{2}\right]^{\frac{1}{2}}-1\right\}\end{array}$ & Degree of change in retardance for linearly polarized light. \\
\hline Linear DOP & $\mathrm{DOP}_{\mathrm{L}}=\frac{m_{10}+m_{11}}{m_{00}+m_{01}}$ & Degree to which linearly polarized input light preserves its polarization state. \\
\hline Circular DOP & $\mathrm{DOP}_{\mathrm{C}}=\frac{m_{30}+m_{33}}{m_{00}+m_{03}}$ & Degree to which circularly polarized input light preserves its polarization state. \\
\hline
\end{tabular}

were chosen based on their sensitivity to sample differences during experiments and their generally intuitive definitions.

\section{Materials and Methods}

\subsection{Sample Preparation}

Porcine tendon and posterior cruciate ligament (PCL) were embedded in optimal cutting temperature compound at $-25^{\circ} \mathrm{C}$. The samples were then cut into thin sections using a cryostat (Leica CM3050S) and soaked in $1 \times$ phosphate-buffered saline to remove the excess embedding compound. Next, the sections were placed on glass microscope slides, and \# 1.5 coverslips were mounted on top with the aqueous mounting media. Tweezers were used to gently lower the coverslip on the microscope slide to avoid creating any air bubbles. After the samples were dried, nail polish was applied on the corners to seal the samples. Note that this study is exempt from the Illinois Institutional Animal Care and Use Committee.

\subsection{Experimental Setup}

Figure 1 shows the SPPARC optical setup. A Ti:Sapphire laser system (Spectra-Physics Mai-Tai) produces 100-fs duration

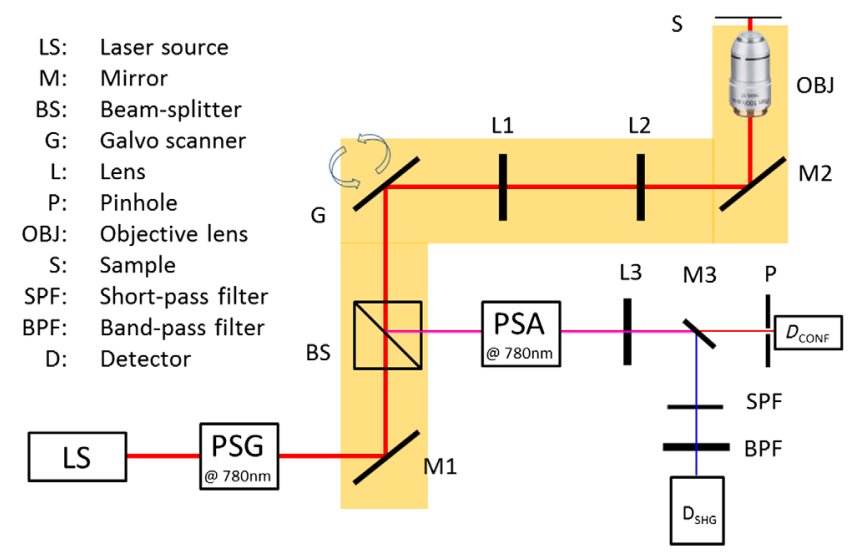

Fig. 1 Experimental setup. The yellow region represents part of the setup that requires critical precalibration. The bold red line shows the path of the illuminating beam. The thin magenta line represents the common path taken by the emitted signals on reflection by the beamsplitter, while the thin red and blue lines show the paths taken by the confocal and SHG signals, respectively. pulses spectrally centered at $780 \mathrm{~nm}$. The polarization state of the input beam is set using a PSG. ${ }^{36}$ This beam is directed through a cube beam-splitter (30:70) and subsequently relayed by mirrors and lenses. A strain-free objective (Olympus ACHN40XP 0.65NA 40X) focuses the beam onto the sample. This relatively lower numerical aperture and strain-free objective is used to allow the focusing behavior to be satisfied within scalar diffraction theory ${ }^{59}$ and to minimize unwanted polarization scattering effects. Scanning galvo mirrors sweep the beam across a $\sim 200-\mu \mathrm{m}$ field-of-view on the sample. The episcattered signal is collected by the same objective, and guided back through the initial path up till the beam-splitter which partially reflects it toward a polarization state analyzer (PSA) ${ }^{36}$ to measure the polarization state of the signal. The beam is focused through a $50-\mu \mathrm{m}$-diameter pinhole at the conjugate plane and then measured by a photomultiplier tube (PMT) detector $D_{\mathrm{CONF}}$ to obtain polarimetric confocal images via different PSG-PSA configurations. SHG images at $390 \mathrm{~nm}$ are obtained using right circularly polarized light and no PSA, by inserting mirror M3 into the beam path. This deflects the beam toward a short-pass (Semrock FF01-680/SP-25) and band-pass (Semrock FF01-390/BP-18-25) filter combination so as to allow only the second-harmonic signal through, which is then detected by the $\operatorname{PMT}\left(D_{\mathrm{SHG}}\right)$.

\subsection{Setup Calibration and Mueller Matrix Characterization}

Polarimetric images obtained using the setup include polarization contributions from the optical setup. In order to isolate the sample's Mueller matrix contribution, a full characterization of the optical setup's Mueller matrix is done. First, the forward matrix $\mathbf{M}_{\mathrm{F}}$ is obtained by PSA-PSG measurements using the arrangement in Fig. 2(a) to be $\mathbf{M}_{\mathrm{F}}=\left(\begin{array}{cccc}1.000 & 0.000 & 0.000 & 0.000 \\ 0.063 & -0.704 & -0.391 & 0.592 \\ -0.083 & -0.675 & 0.644 & -0.396 \\ -0.012 & -0.052 & -0.652 & -0.624\end{array}\right)$.

In a similar manner, the forward + backward matrix $\mathbf{M}_{\mathrm{FB}}$ was obtained from the arrangement in Fig. 2(b) to be $\mathbf{M}_{\mathrm{FB}}=\left(\begin{array}{cccc}1.000 & 0.000 & 0.000 & 0.000 \\ -0.001 & -0.951 & -0.224 & -0.202 \\ 0.039 & 0.144 & 0.179 & -1.000 \\ 0.006 & 0.422 & -0.900 & -0.151\end{array}\right)$. Since we have 


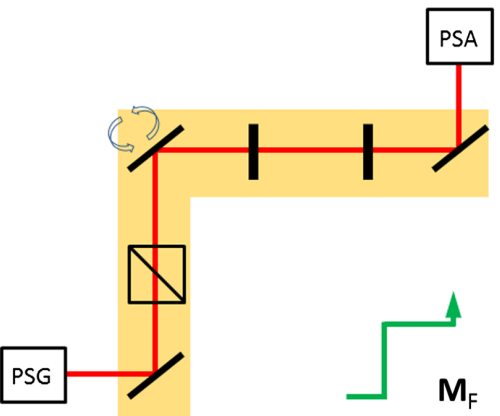

(a)

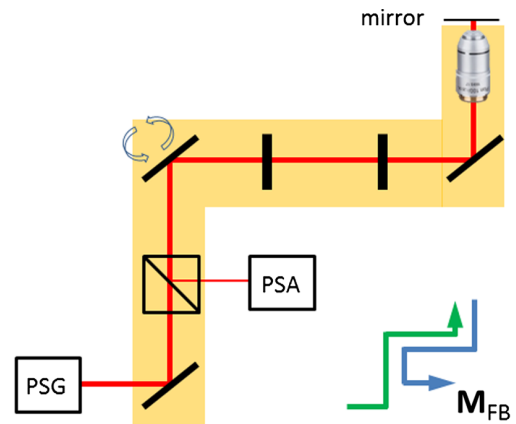

(b)

Fig. 2 Mueller matrix characterization of the optical system. (a) The forward matrix, $\mathbf{M}_{\mathrm{F}}$. (b) The forward + backward matrix, $\mathbf{M}_{\mathrm{FB}}$, using a mirror $\mathrm{M}$ in place of the sample. The arrows to the right bottom corner indicate the path traveled by the beam from generation to analysis.

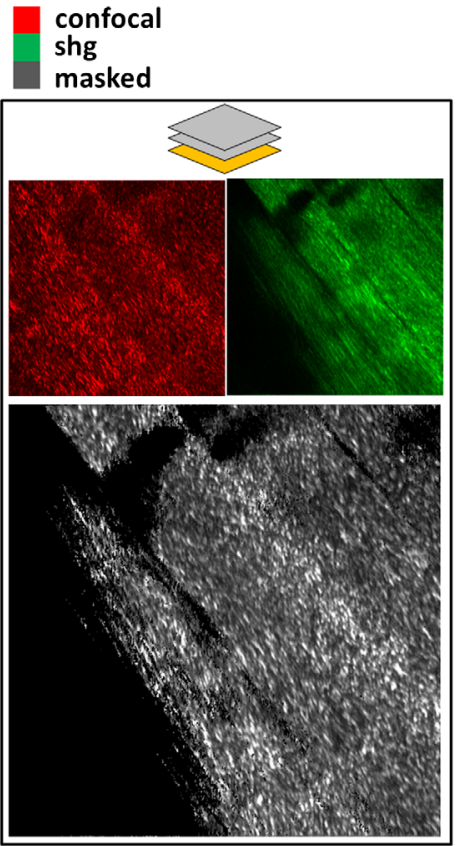

(a)

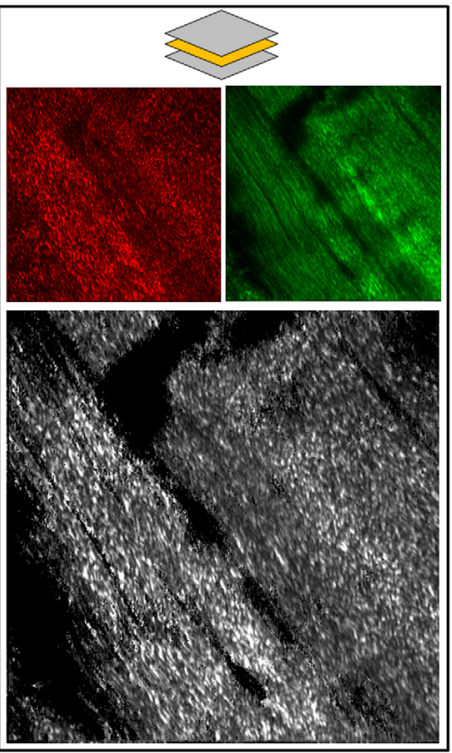

(b)

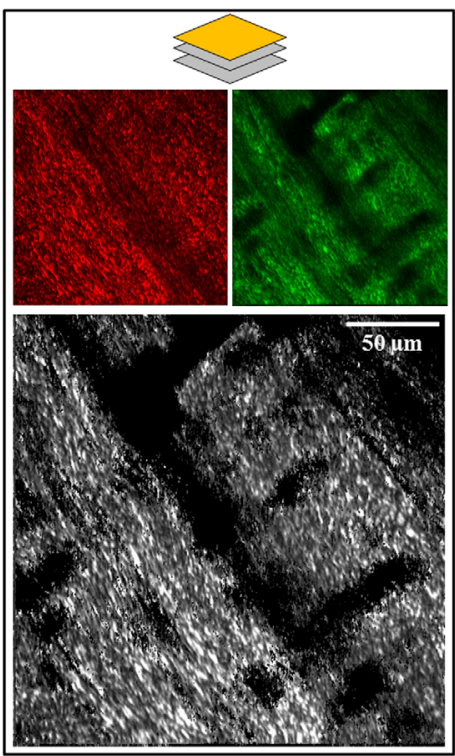

(c)

Fig. 3 (a-c) Confocal (red), SHG (green), and resulting masked (gray) images for three layers of a porcine tendon $z$-stack with a step size of $5 \mu \mathrm{m}$. The currently imaged region in a stack is colored in yellow in the schematic above each column. The $50-\mu \mathrm{m}$ scale bar applies to only the masked images.

$\mathbf{M}_{\mathrm{FB}}=\mathbf{M}_{\mathrm{B}} \mathbf{M}_{\mathrm{mirror}} \mathbf{M}_{\mathrm{F}}$,

the backward matrix $\mathbf{M}_{\mathrm{B}}$ can then be obtained from

$$
\mathbf{M}_{\mathrm{B}}=\mathbf{M}_{\mathrm{FB}}\left(\mathbf{M}_{\mathrm{F}}\right)_{P}^{-1}\left(\mathbf{M}_{\text {mirror }}\right)_{P}^{-1},
$$

where $\mathbf{M}_{\text {mirror }}=\left(\begin{array}{cccc}1 & 0 & 0 & 0 \\ 0 & 1 & 0 & 0 \\ 0 & 0 & -1 & 0 \\ 0 & 0 & 0 & -1\end{array}\right)$ is the ideal mirror Mueller matrix reflecting at normal incidence. ${ }^{60}$ Using $\mathbf{M}_{\mathrm{F}}$ and $\mathbf{M}_{\mathrm{B}}$, the sample matrix $\mathbf{M}_{\text {sample }}$ can be obtained from the measured matrix $\mathbf{M}_{\text {measured }}$ via

$$
\mathbf{M}_{\text {sample }}=\left(\mathbf{M}_{\mathrm{B}}\right)_{P}^{-1} \mathbf{M}_{\text {measured }}\left(\mathbf{M}_{\mathrm{F}}\right)_{P}^{-1}
$$

\subsection{Mueller Matrix Extraction and Decomposition}

For a selected region, 36 polarimetric confocal sample images, from six PSA configurations for each of six PSG states, and a corresponding SHG image are obtained. Each image spans $512 \times 512$ pixels and is collected in $\sim 120 \mathrm{~s}$. The SHG image is binarized by setting a threshold, based on second-harmonic signal strength, to highlight regions with appreciable collagen. This thresholded SHG image is then used as a mask to pattern the polarimetric confocal images. This process has the effect of yielding confocal images of collagen-rich regions. We subsequently determine the Mueller matrix per pixel using an 
image analysis algorithm based on Eq. (3) and extract submatrices and scalar parameters using equations in Table 1.

\section{Results}

Figure 3 shows the confocal (red), SHG (green), and resulting SHG-patterned confocal (grayscale) images for three layers of a 3 -D $z$-stack of porcine tendon, with a step size of $5 \mu \mathrm{m}$. From theoretical considerations, ${ }^{50}$ the axial resolutions are calculated to be $1.4 \cdot n \cdot \lambda_{\mathrm{em} 1} / \mathrm{NA}^{2}(=2.6 \mu \mathrm{m})$ for confocal microscopy, and $2.3 \cdot n \cdot \lambda_{\mathrm{em} 2} / \mathrm{NA}^{2}(=2.1 \mu \mathrm{m})$ for SHG microscopy. The emission wavelengths, $\lambda_{\mathrm{em} 1}$ and $\lambda_{\mathrm{em} 2}$, are given to be 780 and $390 \mathrm{~nm}$, respectively, and $n$ is assumed to be that of air. These values are sufficiently close to show that the images are roughly representative of similar planes. Each slice should also exclude

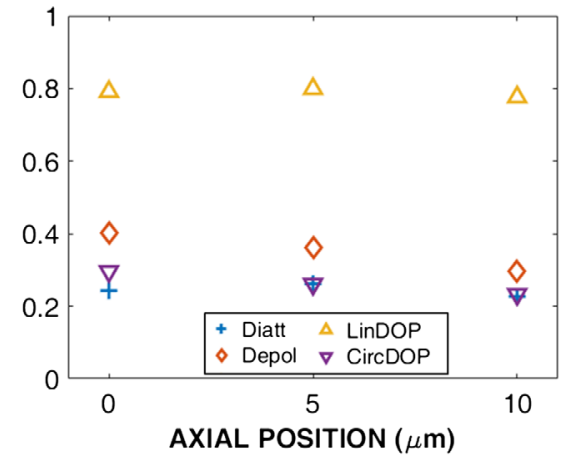

(a)

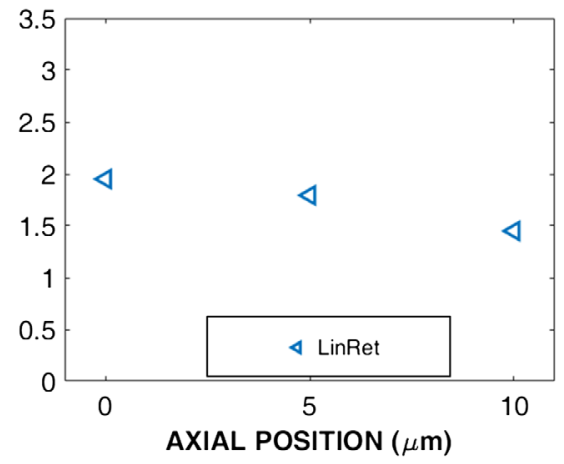

(b)

Fig. 4 Variation of mean polarization parameters for collagen across three porcine tendon layers. (a) Diattenuation, depolarization, linear DOP, and circular DOP, over a 0-to-1 range and (b) linear retardance, over a 0 -to- $\pi$ range. The legend shows the color assignment for each parameter.
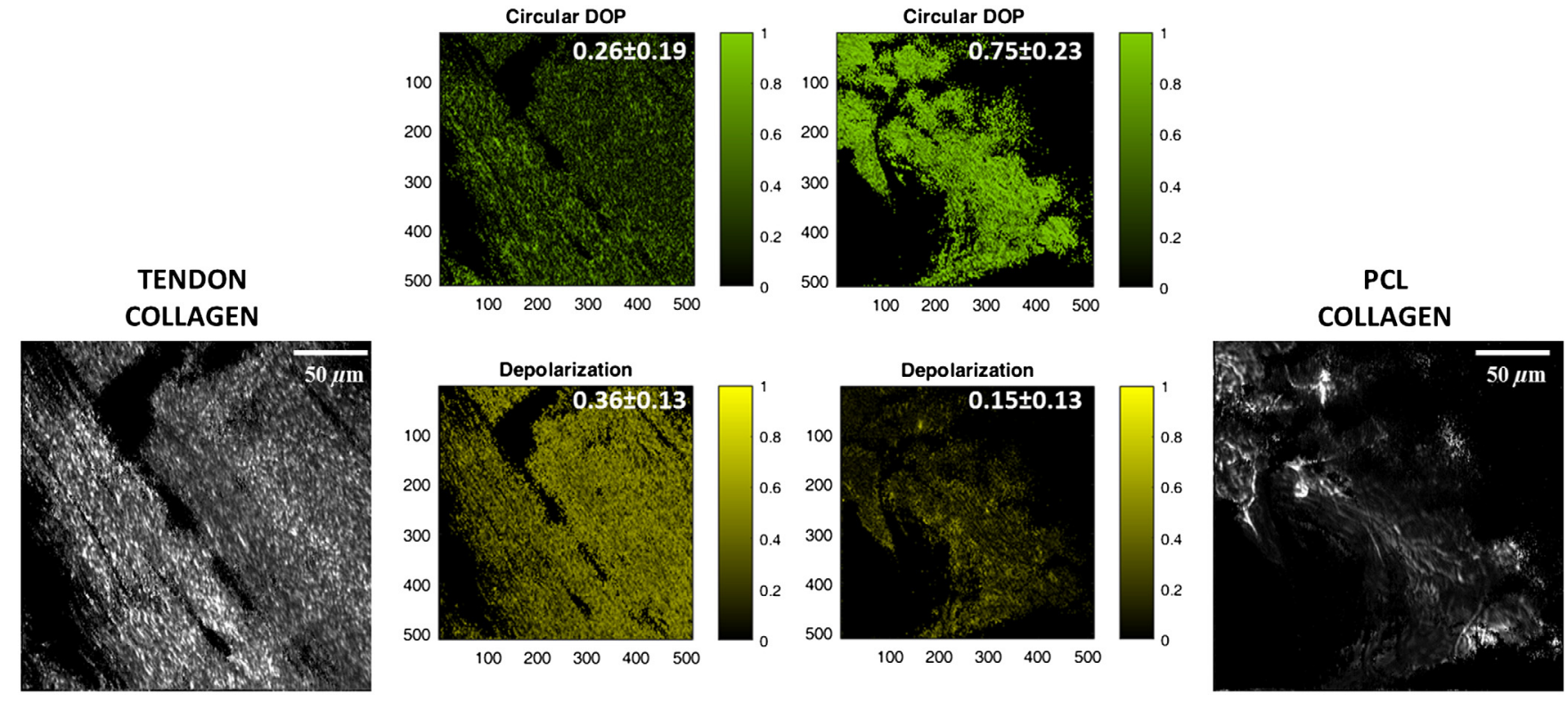

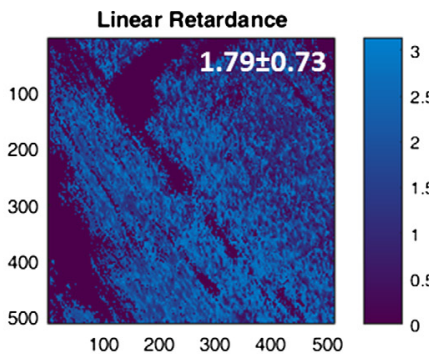

(a)

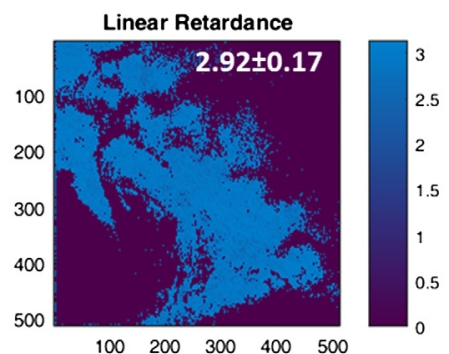

(b)

Fig. 5 Comparison between selected polarization parameter maps (circular DOP, depolarization, and circular retardance) of collagen in porcine (a) tendon and (b) PCL. The color bars represent the range of allowed values for each polarization metric. The inset values at the top right hand of the images are the average parameter values per pixel that contributes to signal, along with the standard deviation. 
significant out-of-plane signal contamination, since the resolution is less than the 5- $\mu \mathrm{m}$ layer separation.

The variation of parameters across the three layers imaged is shown in Fig. 4. From Fig. 4(a), depolarization and circular DOP parameters show a similar trend, reducing in value with deeper tissue penetration. Diattenuation and linear DOP show minimal variation across layers. The high linear DOP values indicate that there is a cumulative effect of preserving linear polarization, which is expected to be along the direction of the fibrils. In Fig. 4(b), it is observed that the average linear retardance reduces with deeper penetration.

Figure 5 shows polarization "heat" map comparisons between selected parameters from collagen in PCL and the center $z$-slice of porcine tendon imaged. On average, PCL has higher circular DOP values (0.75) than tendon (0.25). This implies that circularly polarized light preserves its polarization to a higher degree on passing through PCL. This observation is supported by the depolarization map, since the reduction of circular DOP most likely contributes to the overall depolarization of the sample. Ligament and tendon are connective tissues that have parallel and tightly packed heterogeneous collagen fibers. ${ }^{61}$ However, ligament may also include collagen bundles with spiral arrangement, ${ }^{62}$ and this additional organization variation potentially explains the preferential response to circularly polarized light. Furthermore, the linear retardance of tendon collagen is less than that of PCL collagen. Since linear retardance is related to the birefringence of the sample, this seems to infer that the PCL region imaged has higher birefringence than tendon, which is another expected effect of a spiral/ helical fiber arrangement. ${ }^{63}$

Figure 6 shows the "heat" map comparisons for some parameters from a collagen-rich region and noncollagenous EFMC in PCL. The differences in mean values are more subtle in this case. Also, the polarization data do not show as much variation as the confocal images, and this may be due to additional isotropic absorption which varies across the region imaged and affects intensity but not polarization information. It is observed that, on average, the linear DOP is preserved more for PCL collagen than PCL EFMC. This suggests that there are more components that scatter linear polarization in the EFMC than in fibers. Furthermore, the higher average linear retardance points to the relatively higher birefringence of collagen compared with EFMC. A previous study that alludes to this showed that increased presence of other EFMC components such as proteoglycan caused lower linear birefringence, ${ }^{64}$ inferring that the EFMC has an average lower linear retardance than collagen.
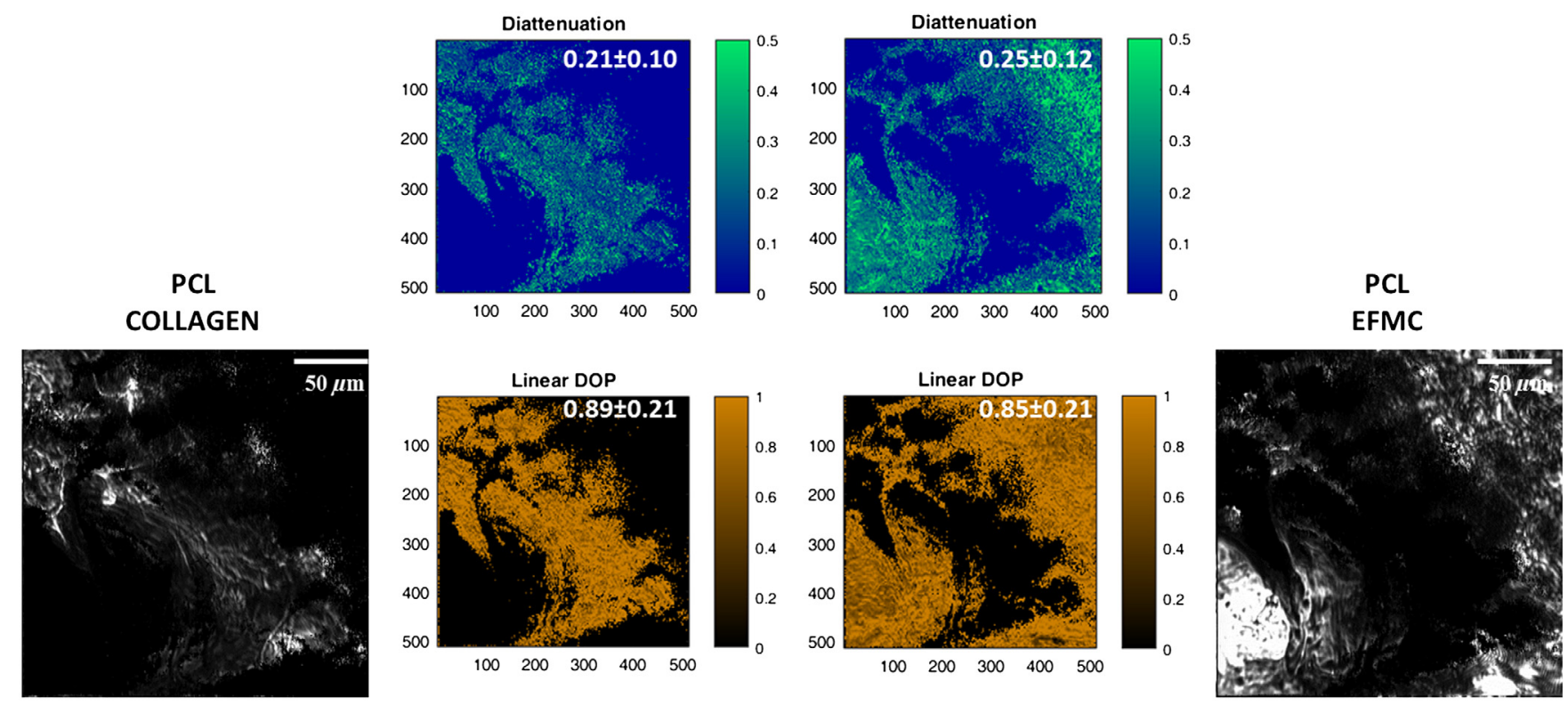

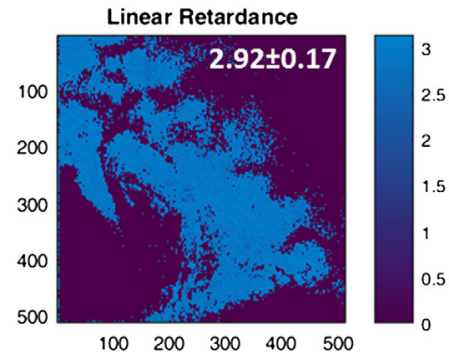

(a)
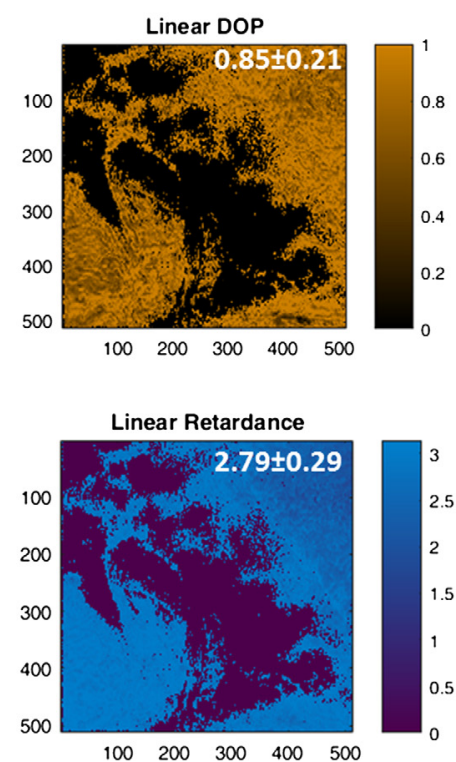

(b)

Fig. 6 Comparison between selected polarization parameter maps (diattenuation, linear DOP, and linear retardance) for (a) collagen and (b) EFMC, in porcine PCL. The color bars represent the range of allowed values for each polarization metric, apart from diattenuation, whose range has a maximum at 0.5 to increase contrast. The inset values at the top right hand of the images are the average parameter values per pixel that contributes to signal, along with the standard deviation. 
However, care must be taken in comparing these values, as the overlapping error bars suggest that the observed differences are not statistically significant.

\section{Summary and Discussion}

In this paper, we introduced the multimodal SPPARC microscope, from which 3-D SHG-patterned confocal microscopy images of collagen fibers were obtained. We applied linear polarimetric analysis and matrix decomposition methods to these images in order to obtain rich polarization information. We also showed that SPPARC microscopy permits label-free quantitative analysis of the EFMC, which to our knowledge had heretofore not been accomplished. Using MMPD as a decomposition approach, we highlighted differences in average parameter values between tendon and PCL collagen and observed that PCL preserves circular DOP (and hence has less total depolarization) than tendon collagen. The variation in parameters was not as pronounced for the comparison between tendon collagen and tendon EFMC. We also tracked changes in parameters across layers for collagen in tendon. The retardance parameters in particular showed gradually reducing values for deeper penetration.

In order to make statistically significant comparisons with stronger interpretation of results, an in-depth study involving more samples and further data analysis would be needed. In addition, the potential of polarimetric measures for differentiation would be more readily realized for complex microstructures having greater diversity, such as the case with varying pathologies of cancerous tissues. This is indeed the focus of ongoing work being undertaken. Furthermore, more recent decomposition methods can be adapted to SPPARC microscopy; one such method is the differential matrix decomposition, ${ }^{65}$ which assumes the sample's properties within a differential matrix set by the spot size of the probing beam.

\section{Disclosures}

The authors have no relevant financial interests in this article and no potential conflicts of interest to disclose.

\section{Acknowledgments}

We thank Woowon Lee for preparing the samples imaged in the experiments.

\section{References}

1. R. Dalgleish, "The human type I collagen mutation database," Nucleic Acids Res. 25(1), 181-187 (1997).

2. G. A. Di Lullo et al., "Mapping the ligand-binding sites and diseaseassociated mutations on the most abundant protein in the human, type I collagen," J. Biol. Chem. 277(6), 4223-4231 (2002).

3. R. G. Wells, "The role of matrix stiffness in regulating cell behavior," Hepatology 47(4), 1394-1400 (2008).

4. P. P. Provenzano et al., "Collagen reorganization at the tumor-stromal interface facilitates local invasion," BMC Med. 4(1), 38 (2006).

5. R. Ambekar et al., "Quantitative second-harmonic generation microscopy for imaging porcine cortical bone: comparison to SEM and its potential to investigate age-related changes," Bone 50(3), 643-650 (2012).

6. A. Gosain and L. A. DiPietro, "Aging and wound healing," World J. Surg. 28(3), 321-326 (2004).

7. G. D. O'Connell, H. L. Guerin, and D. M. Elliott, "Theoretical and uniaxial experimental evaluation of human annulus fibrosus degeneration," J. Biomech. Eng. 131(11), 111007 (2009).
8. S. P. Lake and V. H. Barocas, "Mechanical and structural contribution of non-fibrillar matrix in uniaxial tension: a collagen-agarose co-gel model," Ann. Biomed. Eng. 39(7), 1891-1903 (2011).

9. R. Martin et al., "Collagen fiber organization is related to mechanical properties and remodeling in equine bone. A comparison of two methods," J. Biomech. 29(12), 1515-1521 (1996).

10. E. Brown et al., "Dynamic imaging of collagen and its modulation in tumors in vivo using second-harmonic generation," Nat. Med. 9(6), 796 (2003).

11. M. Winkler et al., "Three-dimensional distribution of transverse collagen fibers in the anterior human corneal stromacorneal collagen fiber angle quantification," Invest. Ophthalmol. Vis. Sci. 54(12), 72937301 (2013).

12. P. Stoller et al., "Quantitative second-harmonic generation microscopy in collagen," Appl. Opt. 42(25), 5209-5219 (2003).

13. Y. Sun et al., "Investigating mechanisms of collagen thermal denaturation by high resolution second-harmonic generation imaging," Biophys. J. 91(7), 2620-2625 (2006).

14. X. Chen et al., "Second harmonic generation microscopy for quantitative analysis of collagen fibrillar structure," Nat. Protoc. 7(4), 654-669 (2012).

15. B. R. Masters et al., Handbook of Biomedical Nonlinear Optical Microscopy, Oxford University Press, New York (2008).

16. R. M. Williams, W. R. Zipfel, and W. W. Webb, "Interpreting secondharmonic generation images of collagen I fibrils," Biophys. J. 88(2), 1377-1386 (2005).

17. R. A. R. Rao, M. R. Mehta, and K. C. Toussaint, "Fourier transformsecond-harmonic generation imaging of biological tissues," Opt. Express 17(17), 14534-14542 (2009).

18. M. Sivaguru et al., "Quantitative analysis of collagen fiber organization in injured tendons using Fourier transform-second harmonic generation imaging," Opt. Express 18(24), 24983-24993 (2010).

19. T. Y. Lau, R. Ambekar, and K. C. Toussaint, "Quantification of collagen fiber organization using three-dimensional Fourier transform-secondharmonic generation imaging," Opt. Express 20(19), 21821-21832 (2012).

20. P. Whittaker et al., "Quantitative assessment of myocardial collagen with picrosirius red staining and circularly polarized light," Basic Res. Cardiol. 89(5), 397-410 (1994).

21. P. G. Ellingsen et al., "Mueller matrix three-dimensional directional imaging of collagen fibers," J. Biomed. Opt. 19(2), 026002 (2014).

22. L. Rich and P. Whittaker, "Collagen and picrosirius red staining: a polarized light assessment of fibrillar hue and spatial distribution," Braz. J. Morphol. Sci. 22(2), 97-104 (2005).

23. R. Oldenbourg and G. Mei, "New polarized light microscope with precision universal compensator," J. Microsc. 180(2), 140-147 (1995).

24. A. Glazer, J. Lewis, and W. Kaminsky, "An automatic optical imaging system for birefringent media," Proc. R. Soc. A 452(1955), 2751-2765 (1996).

25. J. Rieppo et al., "Practical considerations in the use of polarized light microscopy in the analysis of the collagen network in articular cartilage," Microsc. Res. Tech. 71(4), 279-287 (2008).

26. E. M. Spiesz, W. Kaminsky, and P. K. Zysset, "A quantitative collagen fibers orientation assessment using birefringence measurements: calibration and application to human osteons," J. Struct. Biol. 176(3), 302-306 (2011).

27. M. C. Van Turnhout, S. Kranenbarg, and J. L. van Leeuwen, "Modeling optical behavior of birefringent biological tissues for evaluation of quantitative polarized light microscopy," J. Biomed. Opt. 14(5), 054018 (2009).

28. A. Erikson et al., "Quantification of the second-order nonlinear susceptibility of collagen I using a laser scanning microscope," J. Biomed. Opt. 12(4), 044002 (2007).

29. T. Hompland et al., "Second-harmonic generation in collagen as a potential cancer diagnostic parameter," J. Biomed. Opt. 13(5), 054050 (2008).

30. R. Ambekar et al., "Quantifying collagen structure in breast biopsies using second-harmonic generation imaging," Biomed. Opt. Express 3(9), 2021-2035 (2012).

31. W.-L. Chen et al., "Second harmonic generation $\chi$ tensor microscopy for tissue imaging," Appl. Phys. Lett. 94(18), 183902 (2009). 
32. S. Jiao and L. V. Wang, "Two-dimensional depth-resolved Mueller matrix of biological tissue measured with double-beam polarizationsensitive optical coherence tomography," Opt. Lett. 27(2), 101-103 (2002).

33. S.-Y. Lu and R. A. Chipman, "Interpretation of Mueller matrices based on polar decomposition," J. Opt. Soc. Am. A 13, 1106-1113 (1996).

34. M. Dubreuil et al., "Mueller matrix polarimetry for improved liver fibrosis diagnosis," Opt. Lett. 37, 1061-1063 (2012).

35. E. Du et al., "Mueller matrix polarimetry for differentiating characteristic features of cancerous tissues," J. Biomed. Opt. 19(7), 076013 (2014).

36. C. Okoro and K. C. Toussaint, "Experimental demonstration of twophoton Mueller matrix second-harmonic generation microscopy," J. Biomed. Opt. 21(1), 016011 (2016).

37. Y. Shi, W. McClain, and R. Harris, "An extension of the Mueller scattering matrix to nonlinear light scattering," Chem. Phys. Lett. 205(1), 9195 (1993).

38. M. Samim, S. Krouglov, and V. Barzda, "Nonlinear Stokes-Mueller polarimetry," Phys. Rev. A 93(1), 013847 (2016).

39. M. Samim et al., "Characterization of heterogeneous media using nonlinear Stokes-Mueller polarimetry," J. Opt. Soc. Am. B 33(12), 26172625 (2016).

40. Y. Shi, W. McClain, and R. Harris, "Generalized Stokes-Mueller formalism for two-photon absorption, frequency doubling, and hyperRaman scattering," Phys. Rev. A 49(3), 1999-2015 (1994).

41. M. Samim, S. Krouglov, and V. Barzda, "Double Stokes Mueller polarimetry of second-harmonic generation in ordered molecular structures," J. Opt. Soc. Am. B 32(3), 451-461 (2015).

42. M.-R. Antonelli et al., "Mueller matrix imaging of human colon tissue for cancer diagnostics: how Monte Carlo modeling can help in the interpretation of experimental data," Opt. Express 18(10), 10200-10208 (2010).

43. A. F. Fercher et al., "Optical coherence tomography-principles and applications," Rep. Prog. Phys. 66(2), 239-303 (2003).

44. D. Lara and C. Dainty, "Axially resolved complete Mueller matrix confocal microscopy," Appl. Opt. 45, 1917-1930 (2006).

45. J. F. De Boer et al., "Imaging thermally damaged tissue by polarization sensitive optical coherence tomography," Opt. Express 3(6), 212-218 (1998).

46. M. C. Pierce et al., "Birefringence measurements in human skin using polarization-sensitive optical coherence tomography," J. Biomed. Opt. 9(2), 287-291 (2004).

47. S. D. Giattina et al., "Assessment of coronary plaque collagen with polarization sensitive optical coherence tomography (PS-OCT)," Int. J. Cardiol. 107(3), 400-409 (2006).

48. B. E. Applegate et al., "Polarization-resolved second-harmonic-generation optical coherence tomography in collagen," Opt. Lett. 29(19), 2252-2254 (2004).

49. J. M. Schmitt, "Optical coherence tomography (OCT): a review," IEEE J. Sel. Top. Quantum Electron. 5(4), 1205-1215 (1999).

50. A. Diaspro, "Confocal and two-photon microscopy: foundations, applications and advances," in Confocal and Two-Photon Microscopy: Foundations, Applications and Advances, A. Diaspro, Eds., p. 576, Wiley-VCH, New York (2001).

51. J. G. Fujimoto, "Optical coherence tomography for ultrahigh resolution in vivo imaging," Nat. Biotechnol. 21(11), 1361-1367 (2003).
52. A. J. Schriefl et al., "Quantitative assessment of collagen fibre orientations from two-dimensional images of soft biological tissues," J. R. Soc. Interface 9(76), 3081-3093 (2012).

53. J. Pawley and B. R. Masters, "Handbook of biological confocal microscopy," Opt. Eng. 35(9), 2765-2766 (1996).

54. C. Longo et al., "Laser skin rejuvenation: epidermal changes and collagen remodeling evaluated by in vivo confocal microscopy," Lasers Med. Sci. 28(3), 769-776 (2013).

55. B. R. Masters et al., "Rapid observation of unfixed, unstained human skin biopsy specimens with confocal microscopy and visualization," J. Biomed. Opt. 2(4), 437-445 (1997).

56. C. Brosseau, Fundamentals of Polarized Light: A Statistical Optics Approach, Wiley-Interscience, New York (1998).

57. M. Bass et al., Handbook of Optics, Vol. 2, McGraw-Hill, New York (2001).

58. W. Wang et al., "Roles of linear and circular polarization properties and effect of wavelength choice on differentiation between ex vivo normal and cancerous gastric samples," J. Biomed. Opt. 19(4), 046020 (2014).

59. S. Pereira and A. Van de Nes, "Superresolution by means of polarisation, phase and amplitude pupil masks," Opt. Commun. 234(1), 119124 (2004).

60. J. L. Pezzaniti and R. A. Chipman, "Mueller matrix scatter polarimetry of a diamond-turned mirror," Opt. Eng. 34(6), 1593-1598 (1995).

61. V. Ottani, M. Raspanti, and A. Ruggeri, "Collagen structure and functional implications," Micron 32(3), 251-260 (2001).

62. L. David et al., "Biomechanics of ligaments and tendons," Exercise Sport Sci. Rev. 6(1), 125-182 (1978).

63. B. de Campos Vidal, "Image analysis of tendon helical superstructure using interference and polarized light microscopy," Micron 34(8), 423432 (2003).

64. P. Whittaker et al., "Quantitative structural analysis of collagen in chordae tendineae and its relation to floppy mitral valves and proteoglycan infiltration," Heart 57(3), 264-269 (1987).

65. S. Kumar et al., "Comparative study of differential matrix and extended polar decomposition formalisms for polarimetric characterization of complex tissue-like turbid media," J. Biomed. Opt. 17(10), 105006 (2012).

Chukwuemeka Okoro is a $\mathrm{PhD}$ candidate in the Department of Electrical and Computer Engineering at the University of Illinois at Urbana-Champaign, working in the Photonics Research of Bio/ Nano Environments (PROBE) Lab. His research involves developing quantitative second-harmonic generation imaging techniques for assessment of biological tissues.

Kimani C. Toussaint, Jr. is an associate professor in the Department of Mechanical Science and Engineering, and an affiliate in the Departments of Electrical and Computer Engineering, and Bioengineering at the University of Illinois at Urbana-Champaign. He directs an interdisciplinary lab which focuses on developing optical techniques for quantitatively imaging collagen-based tissues, and investigating the properties of plasmonic nanostructures for control of near-field optical forces. He is a senior member in SPIE, OSA, and IEEE. 